\title{
Correlations among elliptic flow parameter, impact parameter, and multiplicity
}

\author{
Dai-Mei Zhou ${ }^{1, a}$, Yun Cheng ${ }^{1,2,5}$, Yu-Liang Yan ${ }^{3}$, Bao-Guo Dong ${ }^{3}$, Ben-Hao Sa ${ }^{1,3,4, b}$, and Laszlo P. Csernai ${ }^{2,5, c}$ \\ 1 Institute of Particle Physics, Huazhong Normal University, 430082 Wuhan, China \\ 2 Institute of Physics and Technology, University of Bergen, Allegaten 55, 5007 Bergen, Norway \\ 3 China Institute of Atomic Energy, P. O. Box 275 (18), 102413 Beijing, China \\ 4 CCAST (World Lab.), P. O. Box 8730, 100080 Beijing, China \\ ${ }^{5}$ Frankfurt Institute for Advanced Studies, Johann Wolfgang Goethe University, Ruth-Moufang-Str. 1, 60438 Frankfurt \\ am Main, Germany
}

Received: 5 May 2010 / Revised: 14 June 2010

Published online: 30 July 2010

(C) The Author(s) 2010. This article is published with open access at Springerlink.com

Communicated by T.S. Bíró

\begin{abstract}
The elliptic flow parameter, $v_{2}$, in the Fourier expansion of the event-by-event charged-particle multiplicity azimuthal distribution in the momentum space is studied by taking into account the multiplicity fluctuations. The correlations among measurable variables of $v_{2}$, charged multiplicity, and impact parameter are investigated using a multiple phase transport model with string melting. It turned out that the charged multiplicity and impact parameter are negatively correlated. This correlation results in the peak structure in $v_{2}$ as a function of the participant nucleon number (charged multiplicity, impact parameter) measured in RHIC experiments. It is suggested that $v_{2}$ calculated in the event-wise average is about $20 \%$ larger than in the particle-wise average, so event-wise average should be used in the theoretical calculations.
\end{abstract}

The charged particles emitted from the fireball created in relativistic nucleus-nucleus collisions exhibit transverse collective flow. This is represented by the elliptic flow parameter $\left(v_{2}\right)$ and other harmonic parameters $\left(v_{n}\right.$, $n=0,1,3,4, \ldots)$. Those parameters, as Fourier expansion coefficients of produced particle azimuthal distribution in the momentum space, are highly sensitive to the spatial geometry of the created fireball (nuclear overlap region or interaction region).

The expected phase transition to Quark-GluonPlasma (QGP) should have a dramatic effect on those harmonic parameters. The consistency between experimental data of $v_{2}\left(p_{T}\right)$ and $v_{2}(y)$ at mid-rapidity and the corresponding hydrodynamic predictions is regarded as an evidence of the production of partonic matter in the ultra-relativistic nucleus-nucleus collisions $[1,2]$. The elliptic flow of high- $p_{T}$ particles may be related to jet fragmentation and parton energy loss [3], which are usually not included in the hydrodynamic calculations. Such kind of hydrodynamic calculation [4] overestimates $v_{2}\left(p_{T}\right)$ in the $p_{T} \geq 1.5 \mathrm{GeV} / c$ region [5]. This is regarded as an evidence of the strongly coupled QGP formation

\footnotetext{
a e-mail: zhoudm@phy.ccnu.edu.cn

b e-mail: sabh@ciae.ac.cn

c e-mail: csernai@ift.uib.no
}

in the relativistic nucleus-nucleus collisions together with the discovery of jet quenching [6]. So far a lot of experimental data have been published on the collective flow parameters [7-9]. Consequently, microscopic transport model studies are also widely progressing [10-14] as well as abundant hydrodynamic investigations.

According to two well-known pioneering works in this field $[15,16]$, the usual study starts from the triple differential distribution

$$
E \frac{\mathrm{d}^{3} N}{\mathrm{~d}^{3} p}=\frac{1}{2 \pi} \frac{\mathrm{d}^{2} N}{p_{T} \mathrm{~d} y \mathrm{~d} p_{T}}\left[1+\sum_{n=1, \ldots} 2 v_{n} \cos \left[n\left(\phi-\Psi_{r}\right)\right]\right],
$$

where $N$ is the particle multiplicity distribution, $\phi$ stands for the azimuthal angle of the particle, and $\Psi_{r}$ refers to the azimuthal angle of the reaction plane in the momentum space. Then the $n$-th flow harmonics is defined as " $v_{n}=\left\langle\cos \left[n\left(\phi-\Psi_{r}\right)\right]\right\rangle$, where \langle\rangle indicates an average over all particles in all events. For the particle number distribution, the coefficient $v_{1}$ is $\left\langle p_{x} / p_{T}\right\rangle$ and $v_{2}$ is $\left\langle\left(p_{x} / p_{T}\right)^{2}-\left(p_{y} / p_{T}\right)^{2}\right\rangle "$ [16]. This kind of average will be indicated as the particle-wise average, in order to distinguish it from the event-wise average introduced in [17]. Later, this particle-wise average has been widely accepted in theoretical calculations either $[10,12]$. 
The problem of the particle-wise average in a sample with wider multiplicity range is that it does not take the influence of multiplicity (hence impact parameter) in a single event of the sample into account. In [17] the elliptic flow and other harmonics have been re-derived starting from the invariant particle multiplicity distribution in the momentum space. It turned out that the harmonic parameter $v_{n}$ (elliptic flow parameter $v_{2}$ ) is an event-wise average of $\cos (n \phi)$

$$
v_{n}^{e}=\langle\overline{\cos (n \phi)}\rangle_{e v} \quad(n=1,2, \ldots),
$$

where $\overline{\cos (n \phi)}$ denotes the average of $\cos (n \phi)$ over particles in a single event, $\langle\ldots\rangle_{e v}$ means an average over events in a sample, and the superscript " $e$ " stands for the eventwise average. It is an average of $\cos (n \phi)$ first over particles in a single event, then over the events in a sample. Here one has to mention that in theory if the beam direction and impact parameter vectors are fixed at the $p_{z}$ and $p_{x}$ axes, respectively, then the reaction plane is just the $p_{x}-p_{z}$ plane [15]. Therefore the reaction plane azimuthal angle $\left(\Psi_{r}\right)$ in eq. (1) introduced for the extraction of elliptic flow in experiments [16] is zero. Meanwhile, the particle-wise average of $v_{n}$ is also derived in [17]

$$
v_{n}^{p}=\left\langle\overline{\cos (n \phi)} N_{e v}\right\rangle_{e v} /\left\langle N_{e v}\right\rangle_{e v} .
$$

That is obviously different from the event-wise average. Only if the $\overline{\cos (n \phi)}$ is independent of event multiplicity $N_{e v}$ (i.e. if the multiplicity plays no role in the average) the $v_{n}^{p}$ reduces to $v_{n}^{e}$. In fact, the $\overline{\cos (n \phi)}$ and $N_{e v}$ correlate (even negatively correlate) with each other. This is because larger event multiplicity arises from more central collisions (larger overlap region between colliding nuclei) and the larger overlap region, in turn, results in less azimuthal asymmetry. The particle-wise average does not take the influence of event multiplicity into account, thus it is questionable from the physics point of view. Of course, for a very narrow multiplicity bin studied, the particlewise average is not very problematic. However, for the wide multiplicity bin the correction is important.

In experiment, the reaction plane is different event by event. In order to extract the elliptic flow parameter one has to invoke a complex reaction plane identification method [16], or the cumulant method [18], or the Lee-Yang zeroes method [19]. In methods [16] and [19] a quantity has to be first constructed event by event. This quantity is just the event plane in [16] and a generating function in [19]. Then a corresponding average over measured events has to be taken, therefore it is event-wise average in the methods of [16] and [19]. In the 2-particle cumulant method [18], the identification of the reaction plane is not required, although it is possible. It is emphasized in all publications that $v_{2}$ can be evaluated with this method without identifying the reaction plane, and therefore the average should run over particles in all events!

In the expansion process of a nucleus-nucleus collision, the initial spatial asymmetry of the created fireball evolves into an azimuthal asymmetry $\left(v_{n}\right)$ in the transverse-momentum distribution of produced charged particles [9]. This evolution process has to be described precisely by the detailed dynamical models via the observation of the $v_{2}\left(v_{n}\right)$ parameter as a function of the charged multiplicity $N_{c h}$ (impact parameter $b$ ). In this work a multiple phase transport model AMPT with string melting [20] is selected as the dynamical model because the AMPT model with string melting has been used successfully to describe the elliptic flow parameter $[10,12]$. $N_{c h}(b)$ is adopted as the argument. The event-wise average versus the particle-wise average in calculating the elliptic flow parameter introduced in [17] is also studied.

In [21] a mapping relation between theoretical and experimental centrality determinations

$$
b=\sqrt{g} b_{\max }, \quad b_{\max }=R_{P}+R_{T}
$$

is introduced. In the above equation, $g$ stands for the total (geometrical) cross-section percentage (or charged multiplicity percentage) used in the experimental determination of centrality. $R_{P}\left(R_{T}\right)$ refers to the radius of the projectile (target) nucleus, $R_{P}=R_{T} \equiv R$ for the symmetry collision system. Obviously, eq. (4) is a relation between impact parameter and charged multiplicity, with which the impact parameter is measurable via charged multiplicity. Equation (4) explains also the equivalence of using $N_{c h}$ or $b$ as the argument of the $v_{2}$-function.

The AMPT model with string melting [20] is a parton and hadron transport model based on the HIJING model [3]. This AMPT model with string melting consists of four stages of the parton initialization, parton evolution (rescattering), hadronization, and hadron evolution (rescattering).

In the first stage, a nucleus-nucleus collision is decomposed into nucleon-nucleon $(\mathrm{NN})$ collisions according to the collision geometry. A NN collision is described by HIJING with the assumption of string melting. I.e. the excited string (soft component) and minijet partons (hard component), formed in HIJING, are then split into constituent quarks and gluons randomly, while the spectator nucleons are kept surviving. So, one obtains an initial partonic state (quarks, antiquarks, but no gluons) for a nucleus-nucleus collision, besides the spectator nucleons.

The partonic rescattering stage follows the initialization one. In this stage the Zhang parton cascade (ZPC) model [22] is employed to describe the partonic rescattering. In ZPC only elastic scatterings are considered with the $g g \rightarrow g g$ cross-section instead of all elastic interaction cross-sections. However, the binary partonparton cross-sections are calculated assuming thermalization. The Monte Carlo method is used to simulate the parton rescattering until the parton-parton collisions cease.

In the hadronization stage the partons after rescattering are hadronized by a phenomenological coalescence model. In the AMPT with string melting model the number of mesons and number of baryons are assumed to be constant after HIJING. The hadronization is practised randomly.

The dynamics of the consequent hadronic matter is described by a relativistic transport (ART) model [23]. In ART the cross-sections of hadron-hardon collisions and hadronic resonances are considered in detail. 


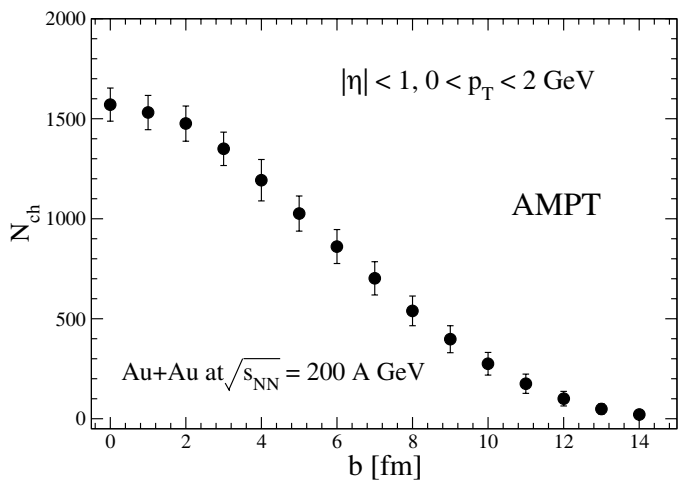

Fig. 1. Correlation between impact parameter $b$ and charged multiplicity $N_{c h}$ calculated by the AMPT model with string melting for the indicated pseudo-rapidity and $p_{T}$ range. The error bars indicate the fluctuations of multiplicity at fixed $b$. Consequently, a fixed event multiplicity may correspond to different $b$.

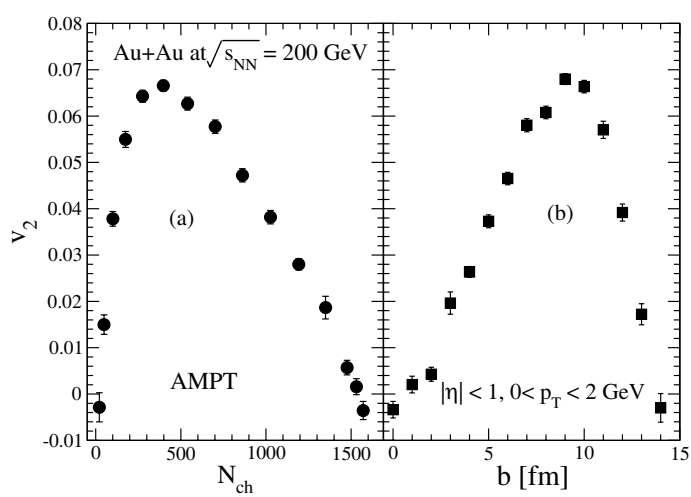

Fig. 2. The integrated $v_{2}$ as a function of $N_{c h}$ (a) and $b(\mathrm{~b})$ calculated by the AMPT model. The error bars indicate the random fluctuation of $v_{2}$ at fixed $N_{c h}$ or $b$.

The AMPT with string melting results given in this paper are calculated by the same code [12] with parameters adjusted to the $v_{2}$ data. The AMPT with string melting model has a realistic description for multiplicity fluctuation at the fixed impact parameter, it is suitable using to check the correlations among measurable variables $v_{2}$, $N_{c h}$, and $b$.

The correlation between $b$ and $N_{c h}$ from the AMPT with string melting calculations for $\mathrm{Au}+\mathrm{Au}$ collisions at $\sqrt{s_{N N}}=200 \mathrm{GeV}$ is shown in fig. 1 . One sees in fig. 1 that $N_{c h}$ is negatively correlated with $b$. A unit of " $\mathrm{fm}$ " change in impact parameter results in more than 50 charged particles change in multiplicity. An about $25 \%$ increase in multiplicity corresponds to about $2 \mathrm{fm}$ decrease in impact parameter.

We give the integrated $v_{2}$ as a function of $N_{c h}(b)$ calculated in the event-wise average by the AMPT model with string melting for the $\mathrm{Au}+\mathrm{Au}$ collisions at $\sqrt{s_{N N}}=$ $200 \mathrm{GeV}$ in fig. 2. Here it has to be mentioned that in the AMPT with string melting publications before this paper, the values of $v_{2}\left(v_{n}\right)$ were calculated with the particlewise average. One sees in fig. 2(a) that a peak appears at $N_{c h}$ nearly equal to 400 . This result is consistent with the PHOBOS and PHENIX reports about the peak struc-

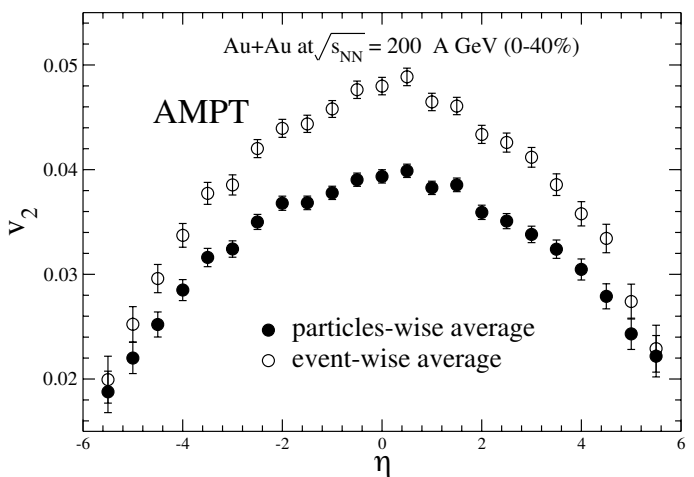

Fig. 3. The integrated $v_{2}$ as a function of $\eta$ calculated by the AMPT model with string melting with particle-wise average average (open circles) and event-wise average (full circles).

ture in $v_{2}$ as a function of the participant nucleon number $\left(N_{\text {part }}\right)$ in $\mathrm{Au}+\mathrm{Au}$ collisions at $\sqrt{s_{N N}}=200 \mathrm{GeV}[5,24]$.

Similarly, we see in fig. 2(b) that the peak of $v_{2}$ appears at $b$ close to the radius of the colliding nucleus $\left(R_{\mathrm{Au}}=7.06 \mathrm{fm}\right)$. This is the result of competition between the charge multiplicity and impact parameter. In central $\mathrm{Au}+\mathrm{Au}$ collisions the charge multiplicity reaches the maximum at zero impact parameter, where the nuclear overlap region is nearly symmetric thus $v_{2}$ approaches zero $[5,9]$. In the middle peripheral collisions, although the charge multiplicity is going down, the $v_{2}$ is large because of the strong asymmetry of the nuclear overlap region (large impact parameter). In the most peripheral collisions, the asymmetry of the nuclear overlap region is very strong, while the $v_{2}$ is going down because the multiplicity is too low to generate a pressure gradient. When the impact parameter is around the radius of the colliding nucleus, the charge multiplicity is not so small, and the asymmetry of the nuclear overlap region is considerably strong, so $v_{2}$ approaches its maximum.

In fig. 1 we see that a nearly $25 \%$ increase in multiplicity corresponds to about $2 \mathrm{fm}$ decrease in the impact parameter. This change in the impact parameter, in turn, results in about a change of 0.015 in $v_{2}$ (see the sensitive region of $2<b<7$ in fig. 2(b)). Thus, the AMPT model with string melting, incorporating random multiplicity fluctuation, verifies that a charge multiplicity bin, which includes, e.g., $25 \%$ of maximum charged multiplicity, spans an impact parameter range of $\Delta b \sim 2 \mathrm{fm}$. This results in a change of $\Delta v_{2} \sim 0.015$ in the AMPT with string melting calculations. Considering the maximum $v_{2}$ in the AMPT with string melting calculations is just 0.068 (cf. fig. 2(b)), so the change of $\Delta v_{2}$ caused by the different impact parameters in the sample is about $20-30 \%$ of the maximum! This is a very significant change, which should not be underestimated!

In addition, we use the AMPT model with string melting to calculate $v_{2}^{e}(\eta)$ and $v_{2}^{p}(\eta)$ in $0-40 \%$ most central $\mathrm{Au}+\mathrm{Au}$ collisions at $\sqrt{s_{N N}}=200 \mathrm{GeV}$. The AMPT with string melting results of $v_{2}^{e}(\eta)$ are nearly $20 \%$ larger than $v_{2}^{p}(\eta)$ as shown in fig. 3. This means $\left\langle\overline{\cos (n \phi)} N_{e v}\right\rangle_{e v}$ is smaller than $\langle\overline{\cos (n \phi)}\rangle_{e v}\left\langle N_{e v}\right\rangle_{e v}$ and demonstrates the negative correlation between $\overline{\cos (n \phi)}$ and $N_{e v}$ again. 
In summary, the physics of how the initial spatial asymmetry evolves into final momentum asymmetry is investigated in AMPT with string melting calculations taking into account the multiplicity fluctuations. The correlations between measurable variables of $v_{2}, N_{c h}$, and $b$ are investigated. It turned out that the impact parameter is negatively correlated with charged multiplicity. This correlation results in the peak structure in $v_{2}$ as a function of $N_{\text {part }}\left(N_{c h}, b\right)$ measured by PHOBOS and PHENIX $[5,24]$. The peak location is approximately corresponding to $b$ being close to the radius of the colliding nucleus.

The averaging procedure in the definition of the elliptic flow parameter is examined by AMPT with string melting calculations for $v_{2}^{e}(\eta)$ and $v_{2}^{p}(\eta)$ in $0-40 \%$ most central $\mathrm{Au}+\mathrm{Au}$ collisions at $\sqrt{s_{N N}}=200 \mathrm{GeV}$. The AMPT with string melting results of $v_{2}^{e}(\eta)$ are about $20 \%$ larger than $v_{2}^{p}(\eta)$. This emphasizes again the necessity of theoretically calculating the elliptic flow parameter $v_{2}$ by the event-wise average.

Financial support from NSFC, China (grants 10705012, 10475032, 10605040, 10635020, 10975062,) RCN, Norway (grants 101937 and 171247), the Chinese-Norwegian RCNNFSC collaboration grant, Nos. 10811130243, 10811130244, and 10811130245, as well as the Programme of Introducing Talents of Discipline to Universities under Grant No. B08033 are gratefully acknowledged. We thank Prof. Lie-Wen Chen for the help in running the AMPT model.

Open Access This article is distributed under the terms of the Creative Commons Attribution Noncommercial License which permits any noncommercial use, distribution, and reproduction in any medium, provided the original author(s) and source are credited.

\section{References}

1. M. Gyulassy, in Proceedings of the NATO ASI on Structure and Dynamics of Elementary Matter, Camyuva-Kemer (Antalya), Turkey, Sep 22 - Oct 2, 2003, edited by W.
Greiner, M.G. Itkis, J. Reinhardt, M. Cem Guclu (Kluwer Academic Publishers, Dordrecht/Boston/London, 2004) ISBN: 1-4020-2445-2, arXiv:nucl-th/0403032.

2. L.P. Csernai, D. Röhrich, Phys. Lett. B 458, 454 (1999).

3. M. Gyulassy, X.-N. Wang, Comput. Phys. Commun. 83, 307 (1994).

4. P.F. Kolb, P. Huovinen, U.W. Heiz, H. Heiselberg, Phys. Lett. B 500, 232 (2001).

5. Phobos Collaboration (B.B. Back et al.), Phys. Rev. C 72, 051901(R) (2005).

6. W.A. Zajc, arXiv:0802.3552.

7. STAR Collaboration (B.I. Abelev), Phys. Rev. Lett. 99, 112301 (2007) and references therein.

8. PHENIX Collaboration (S.S. Adler et al.), Phys. Rev. Lett. 99, 052301 (2007) and references therein.

9. PHOBOS Collaboration (B. Alver et al.), Phys. Rev. Lett. 98, 242302 (2007) and references therein.

10. Bin Zhang, M. Gyulassy, Che-Ming Ko, Phys. Lett. B 455, 45 (1999).

11. E.E. Zabrodin, C. Fuchs, L.V. Bravina, A. Faessler, Phys. Lett. B 508, 184 (2001).

12. Lie-Wen Chen, V. Greco, Che-Ming Ko, P.F. Kolb, Phys. Lett. B 605, 95 (2005).

13. Zhe Xu, C. Greiner, H. Stoeker, Phys. Rev. Lett. 101, 082902 (2008).

14. Xianglei Zhu, M. Bleicher, H. Stöcker, Phys. Rev. C 72, 064911 (2005).

15. S.A. Voloshin, Y. Zhang, Z. Phys. C 70, 665 (1996).

16. A.M. Poskanzer, S.A. Voloshin, Phys. Rev. C 58, 1671 (1998).

17. Xiao-Mei Li, Bao-Guo Dong, Yu-Liang Yan, Hai-Liang Ma, Dai-Mei Zhou, Ben-Hao Sa, Mod. Phys. Lett. A 25, 1211 (2010).

18. N. Borghini, P.M. Dinh, J.-Y. Ollitrault, Phys. Rev. C 63, 054906 (2001).

19. R.S. Bhalerao, N. Borghini, J.-Y. Ollitrault, Nucl. Phys. A $\mathbf{7 2 7}, 373$ (2003).

20. Z.W. Lin, C.M. Ko, B.A. Li, B. Zhang, S. Pal, Phys. Rev. C 72, 064901 (2005).

21. Ben-Hao Sa, A. Bonasera, An Tai, Dai-Mei Zhou, Phys. Lett. B 537, 268 (2002).

22. B. Zhang, Comput. Phys. Commun. 109, 193 (1998).

23. B.A. Li, C.M. Ko, Phys. Rev. C 52, 2037 (1995).

24. PHENIX Collaboration (M. Shimomura), arXiv:0908. $0052 \mathrm{v} 2$. 